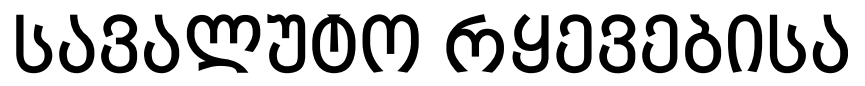

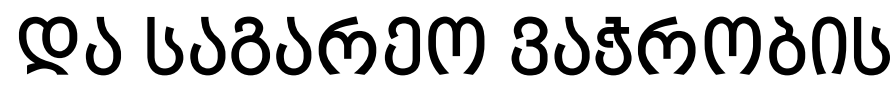

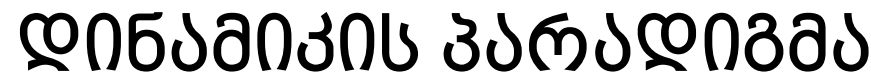

\author{
https://doi.org/10.35945/gb.2016.02.003
}

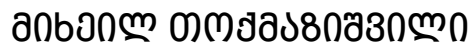

эзm6manz

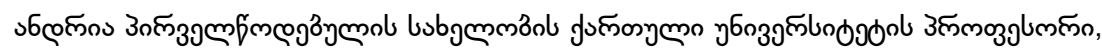

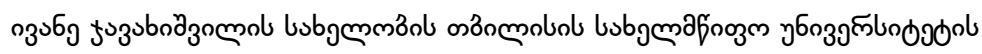

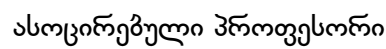

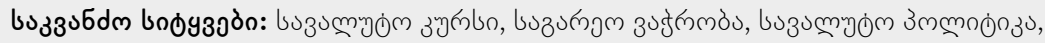

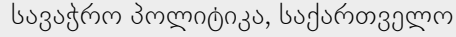

○

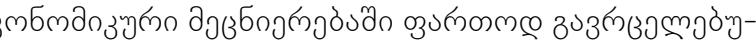

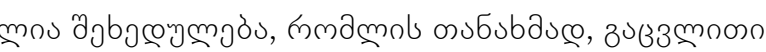

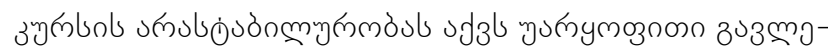

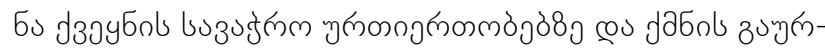

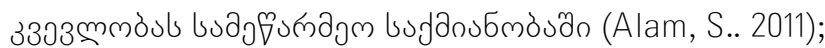

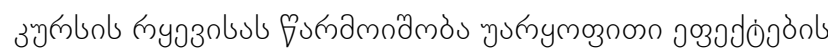

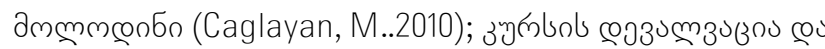

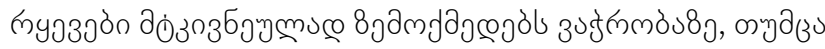

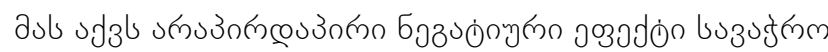

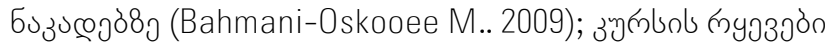

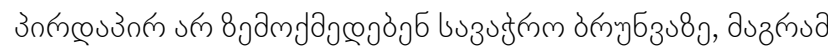

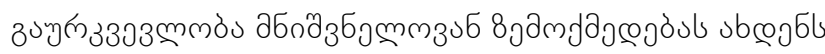

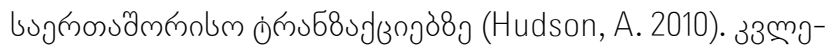

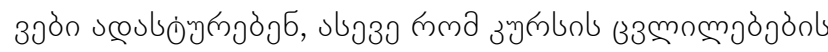

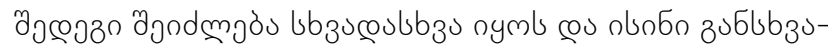

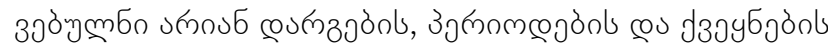
gुbuósanlsuco.

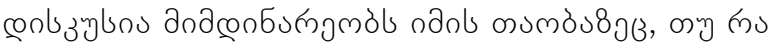

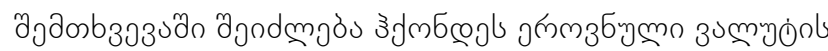

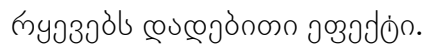

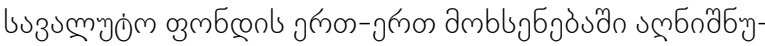

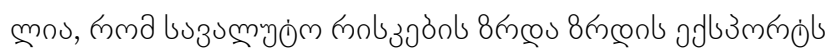

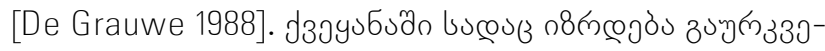

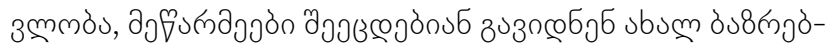

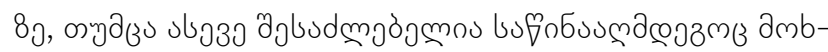

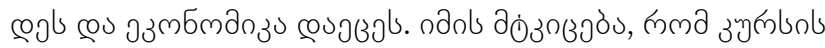

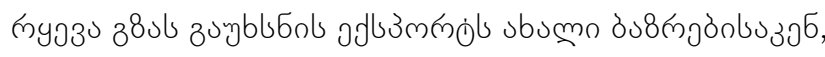

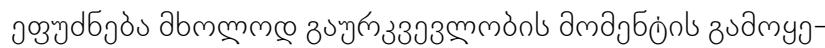

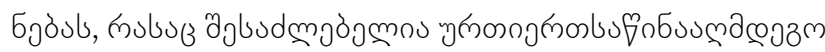

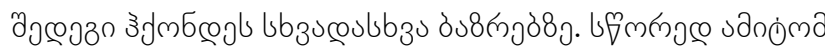

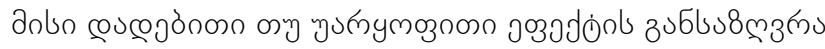

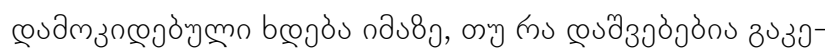

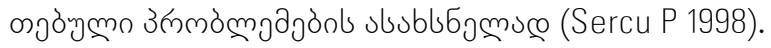

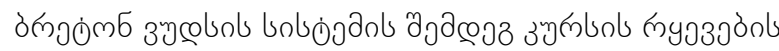

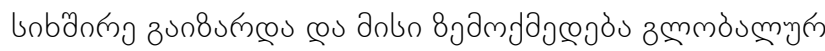

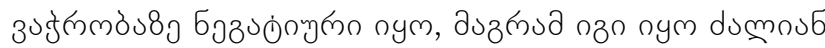

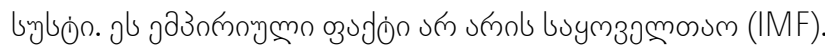

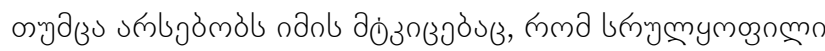

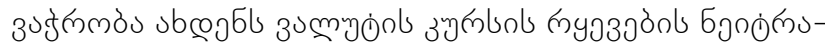

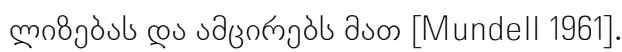

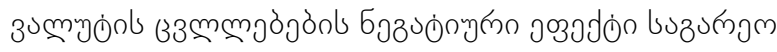

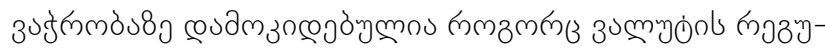

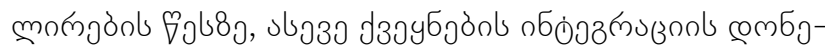

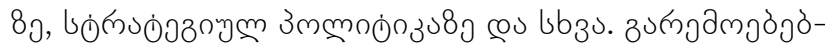

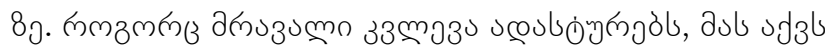

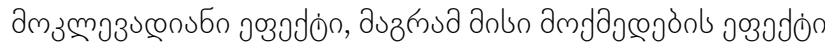

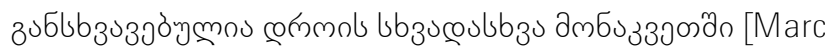

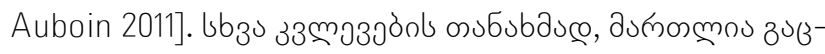

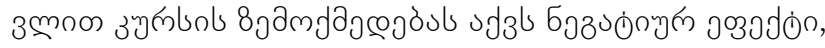

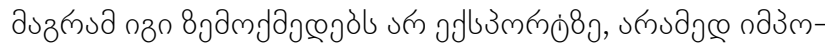

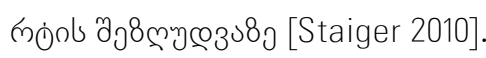

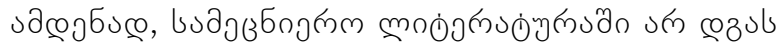




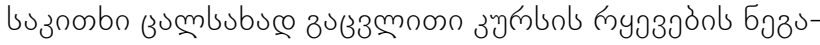

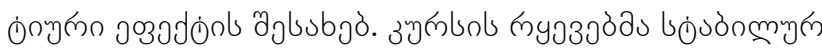

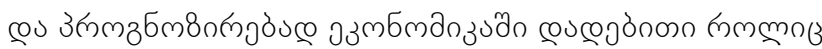

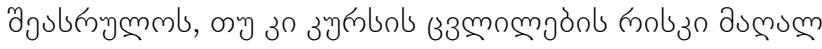

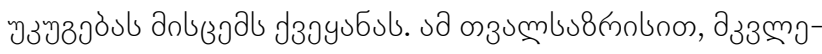

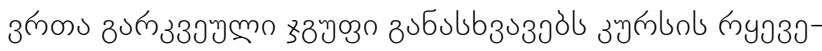

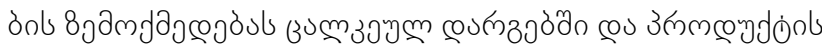

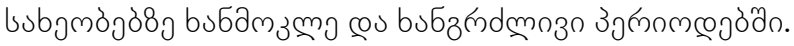

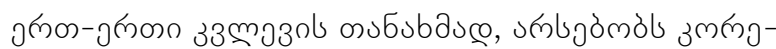

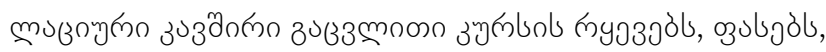

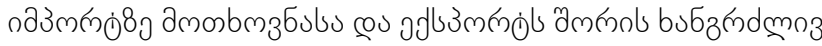

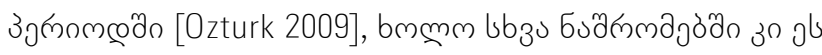

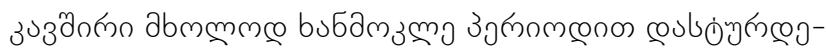

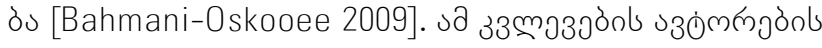

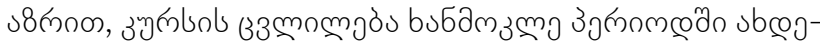

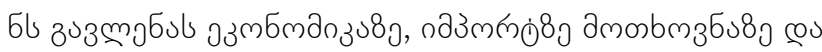

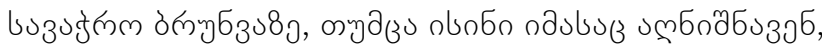

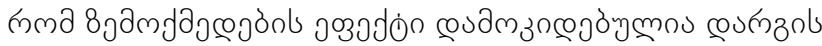

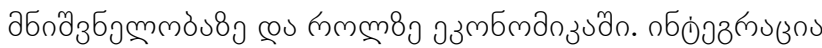

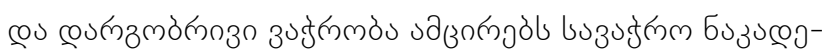

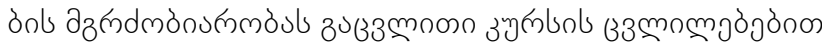

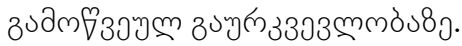

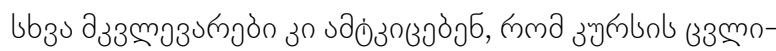

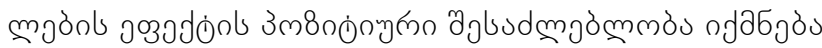

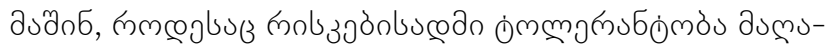

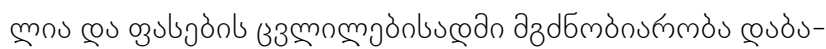

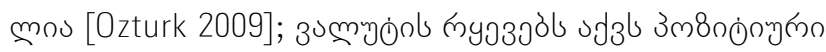

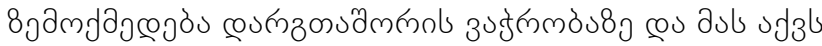

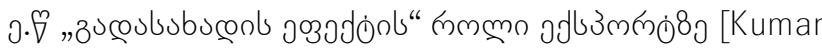

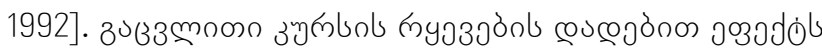

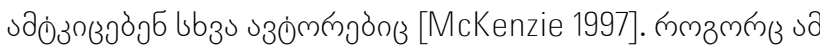

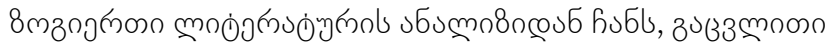
з з

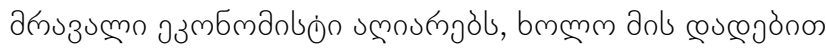

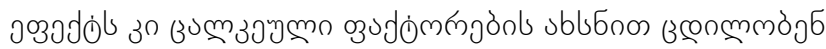
[Krugman 2010:450].

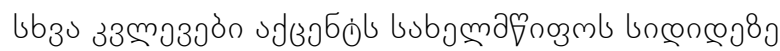

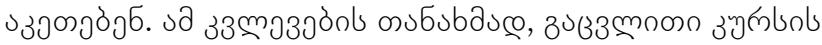

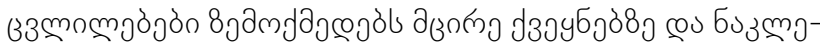

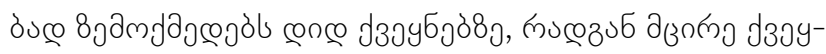

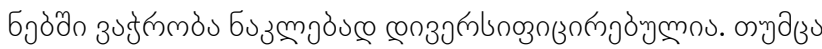

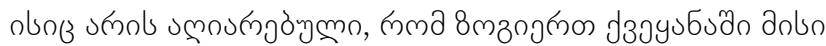

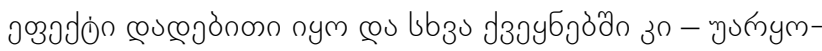

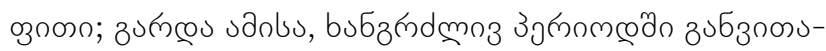

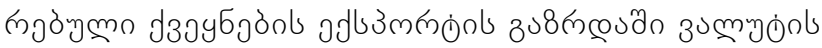

3ง

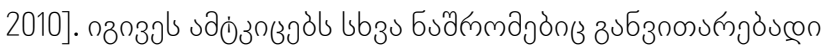
dзoyбjòns aszumnon8o [Haddad 2010].

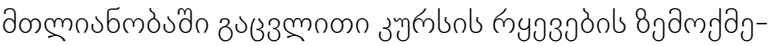

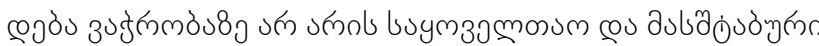

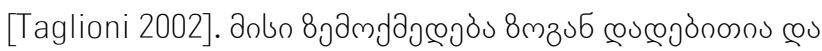

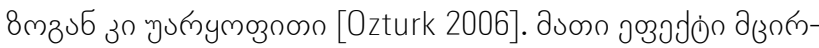

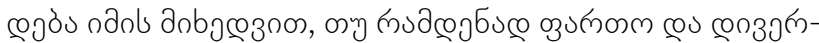

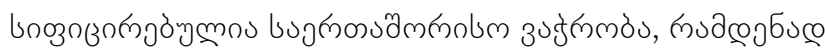

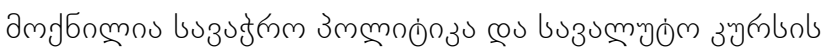

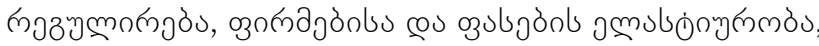

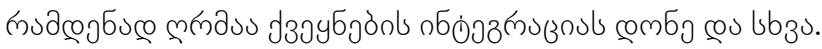

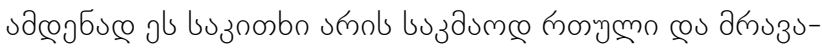

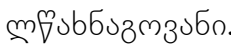

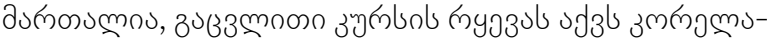

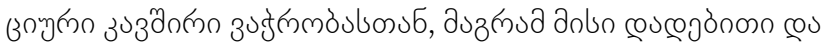

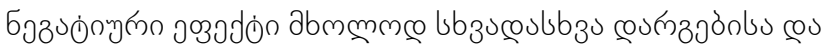

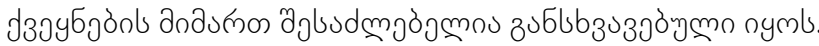

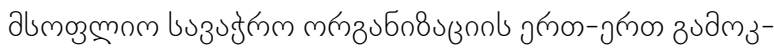

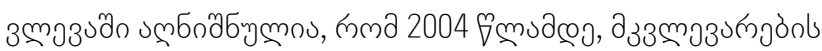

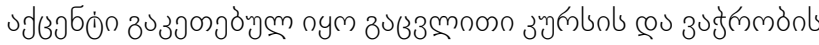

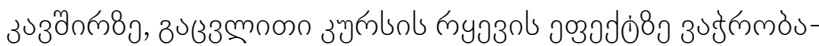

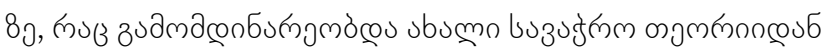
("new-new" trade theory), bmmm 2005 fmmnœub ud (зəбón

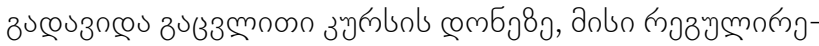

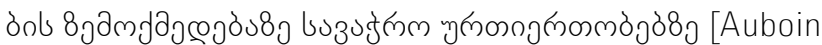
2011].

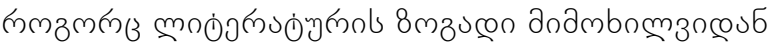

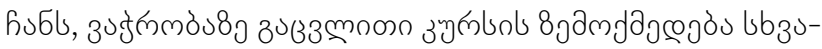

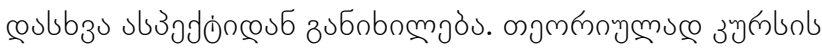

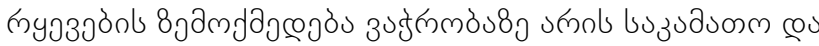

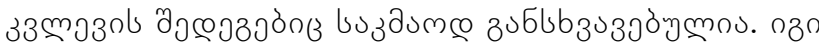

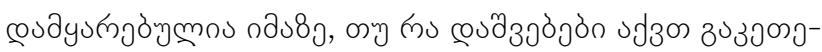

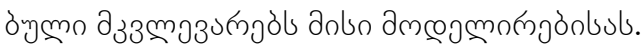

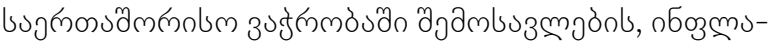

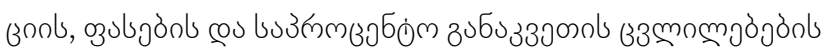

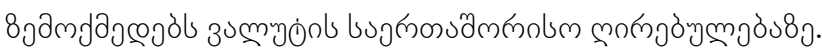

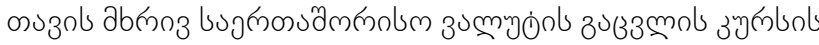

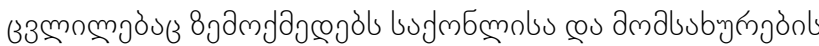

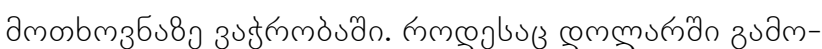

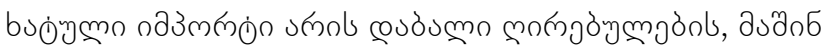

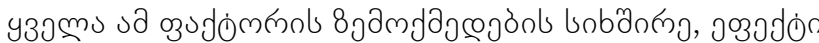

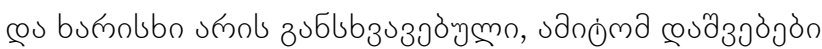

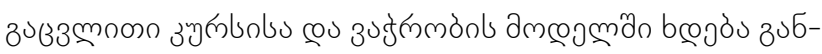

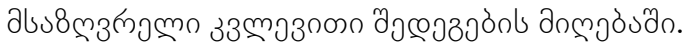




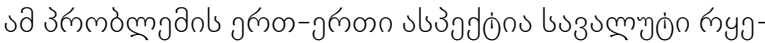

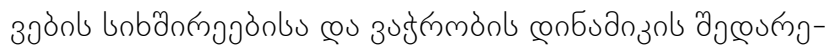

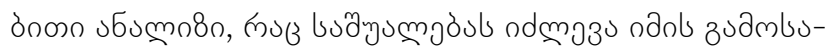

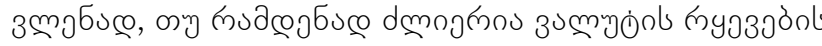

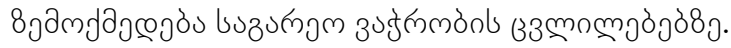

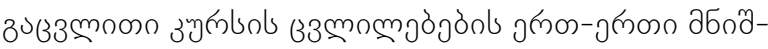

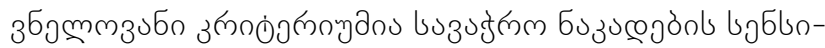

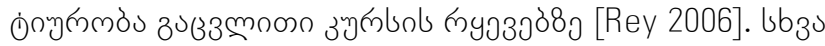

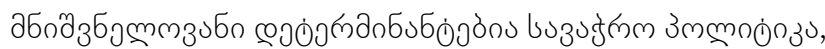

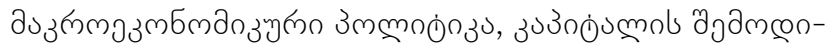

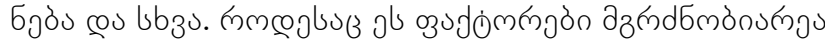

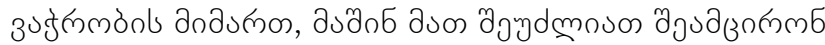

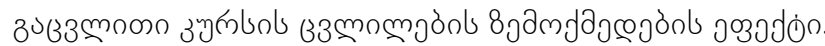

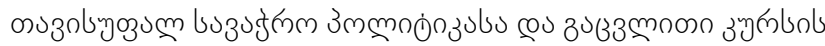

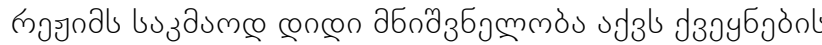

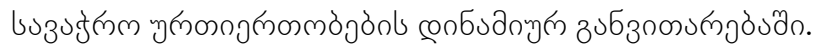

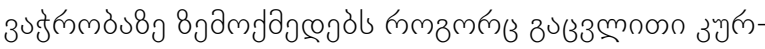

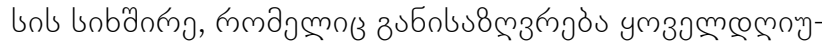

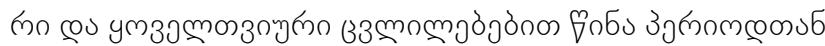

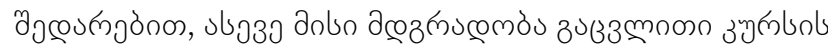

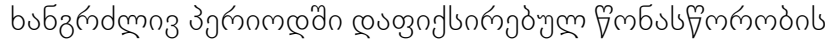

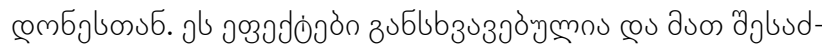

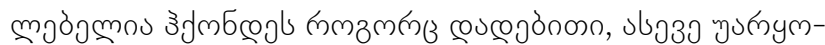

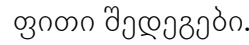

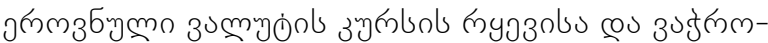

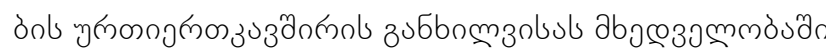
33دdz bا

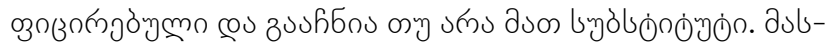

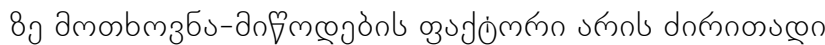

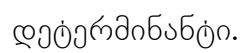

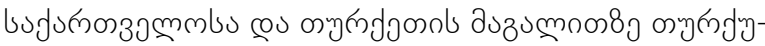

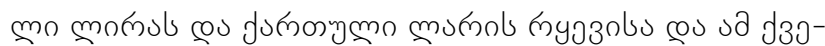

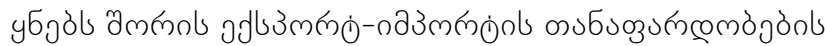

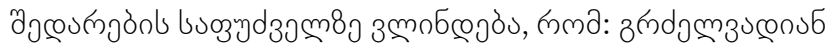

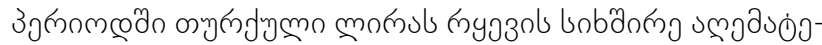

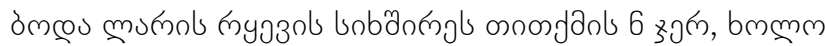

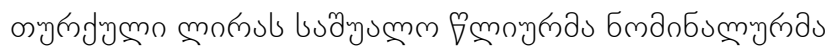

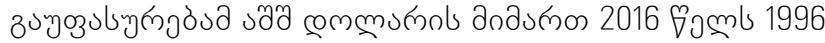

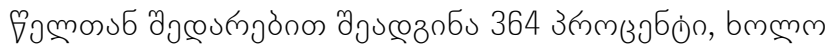

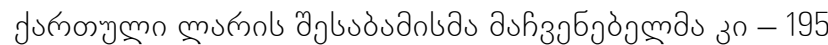

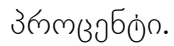

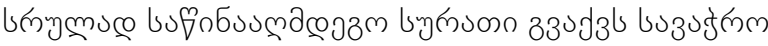

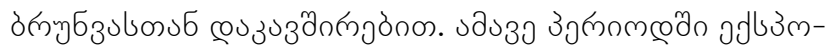

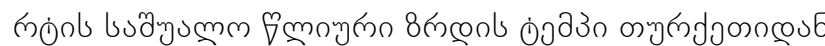

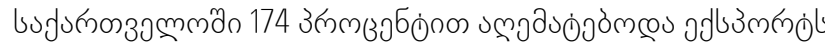

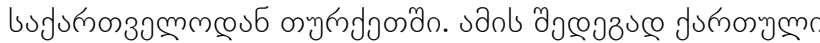

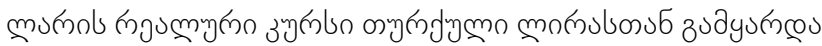

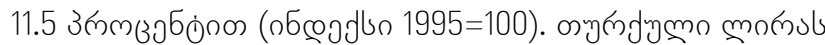

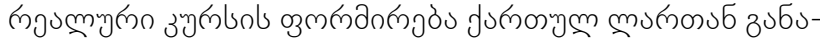

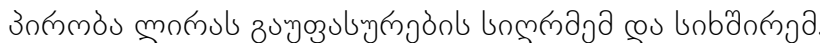

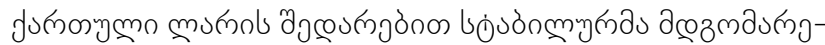

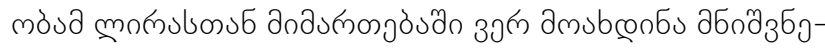

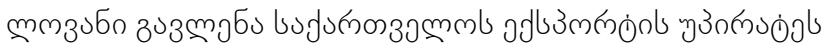

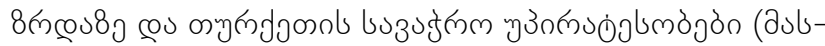

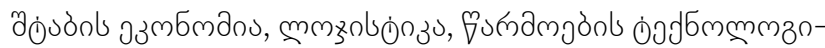

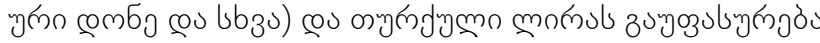

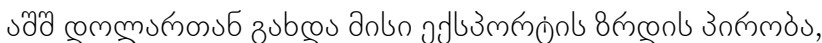

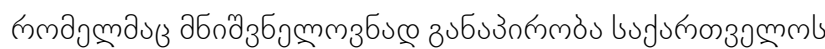

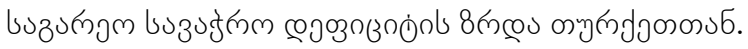

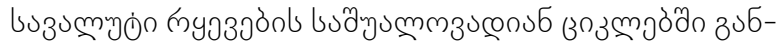

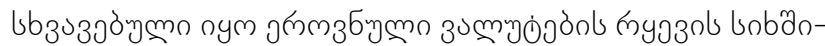

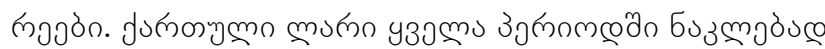

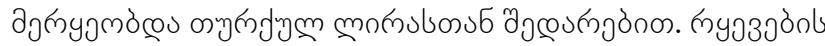

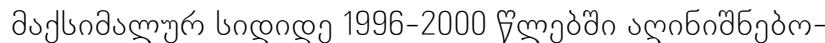

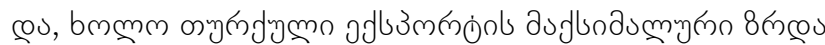

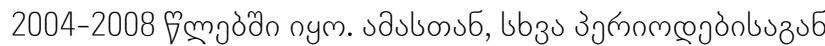

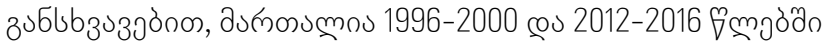

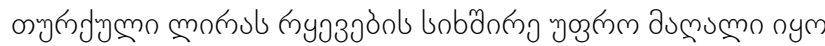

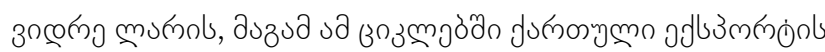

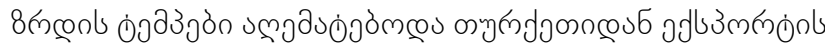

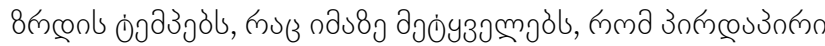

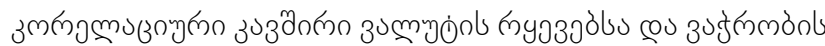

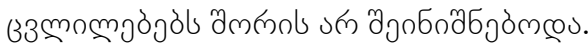

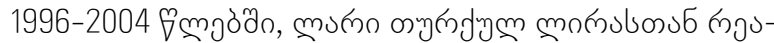

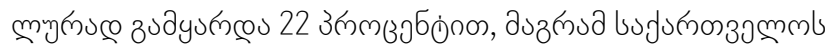

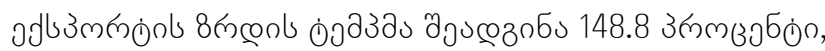

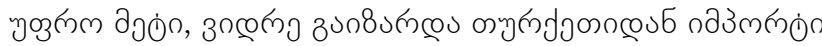

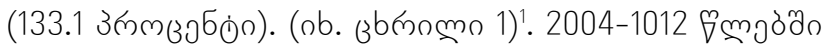

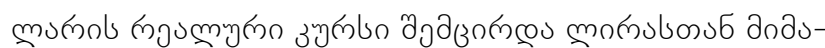

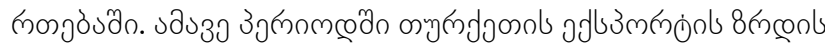

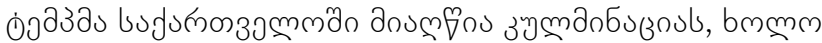

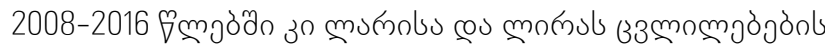

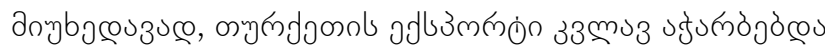

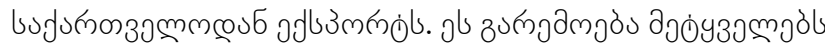

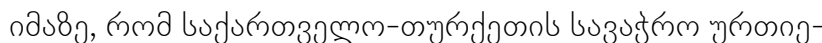

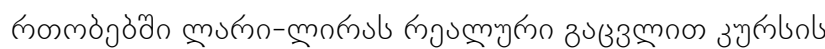

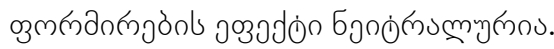

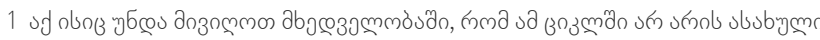

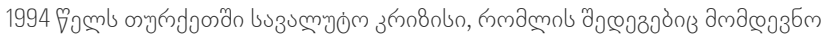

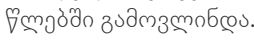




\begin{tabular}{|c|c|c|c|c|c|}
\hline & $1996-2000$ & $2000-2004$ & 2004-2008 & 2008-2012 & 2012-2016 \\
\hline 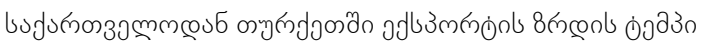 & 115.7 & 133.1 & 128.0 & 100.3 & 100.3 \\
\hline 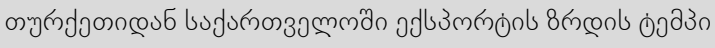 & 105.9 & 125.2 & 154.7 & 116.9 & 102.7 \\
\hline 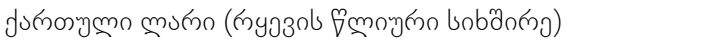 & 0.13 & 0.04 & 0.06 & 0.04 & 0.14 \\
\hline 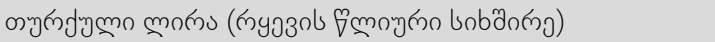 & 0.55 & 0.13 & 0.29 & 0.38 & 0.26 \\
\hline 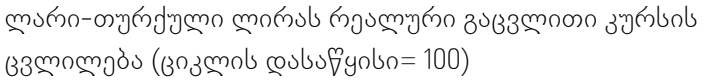 & 80 & 98 & 115 & 126 & 70 \\
\hline
\end{tabular}

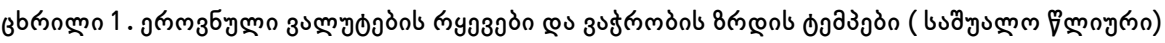

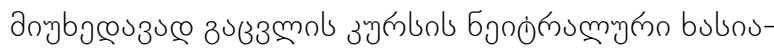

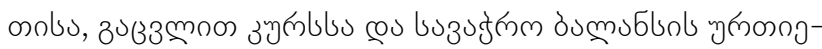

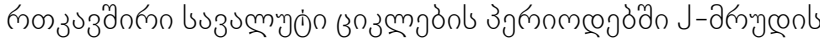

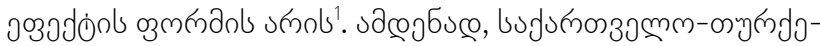

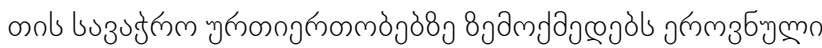

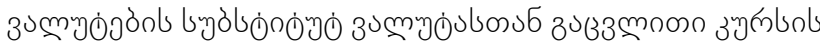
knyozoòn.

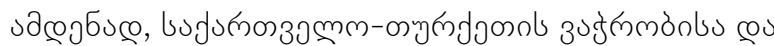

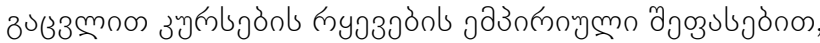

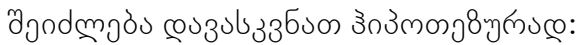

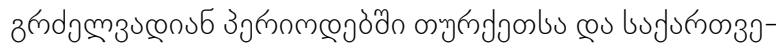

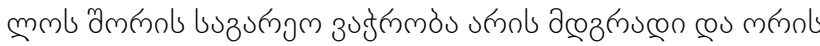

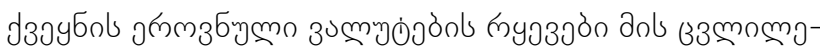

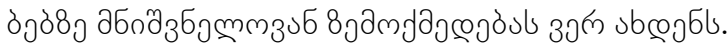

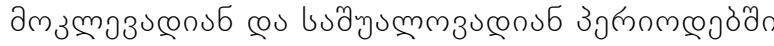

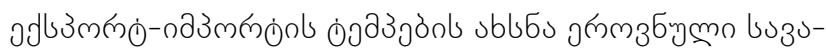

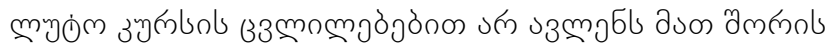

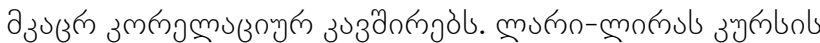

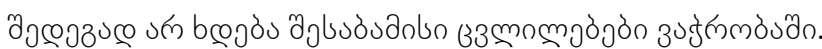

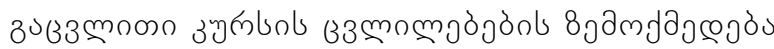

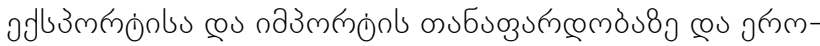

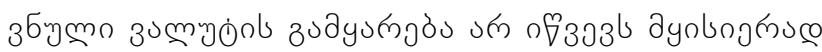

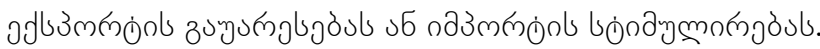

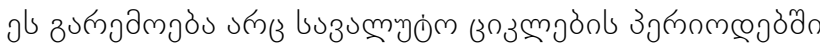
uмnбกวблдे.

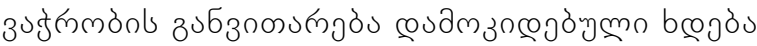

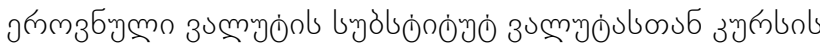

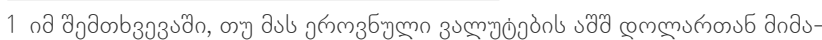

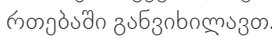

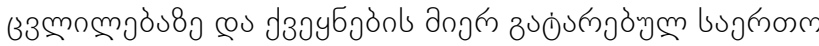

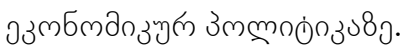

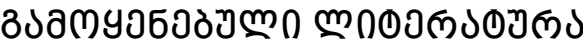

1. Alam, S. And Ahmad, 0. (2011). "Exchange Rate Volatility and Pakistan's Bilatera Imports from Major Sources: An Application of ARDL Approach", International Journal of Economics and Finance, Vol. 3, No. 2.

2. Caglayan, M. and Di, J. (2010) "Does Real Exchange Rate Volatility Affect Sectoral Trade Flows?" Southern Economic Journal 2010, 77(2).

3. Bahmani-Oskooee, M. and Hegerty, S. W. (2009). "The Effects of Exchange-Rate Volatility on Commodity Trade between the United States and Mexico", Southern Economic Journal, 2009, 75(4).

4. Hudson, A. and Straathof, B. (2010) "The Declining Impact of Exchange Rate Volatility on Trade", De Economist 158, No.4, 2010.

5. De Grauwe, P. (1988), "Exchange Rate Variability and the Slowdown in Growth of International Trade", IMF Staff Papers, 35.

6. Sercu, P. and Uppal, R. (1998), "Exchange Rate Volatility, International Trade: A general Equilibrium analysis", Mimeo, University of British Colombia. http://facultyresearch.london.edu/docs/SercuUppal.pdf

7. Exchange Rate Volatility and Trade Flows - Some New Evidences, IMF (2004), Occasional Paper 235.

8. Mundell, Robert (1961), A Theory of Optimum Currency Areas, American Economic Review, 51, (September). https://www.aeaweb.org/aer/top20/51.4.657-665.pdf

9. Auboin Marc and Ruta Michele, The Relationship 
between Exchange rates and International Trade: a Review of Economic Literature. Staff Working Paper ERSD-2011-17 27, WTO, October 2011.

10. Staiger, Robert W. and Alan 0. Sykes (2010), "Currency manipulation" and world trade, World Trade Review 9(4).

11. Ozturk, I. and Kalyonju, H. (2009), "Exchange Rate Volatility and Trade: An Empirical Investigation from CrossCountry Comparison“, African Development Review, Vol. 21, No. 3.

12. Bahmani-Oskooee, M. and Hegerty, S.W. (2009) "The Effects of Exchange-Rate Volatility on Commodity Trade between the United States and Mexico“, Southern Economic Journal, 2009, 75(4).

13. Ozturk, I. and Kalyonju, H. (2009), "Exchange Rate Volatility and Trade: An Empirical Investigation from CrossCountry Comparison“, African Development Review, Vol. 21, No. 3.

14. Kumar, Vikram (1992), The Real Effects of Exchange Rate Risk on International Trade, Working Paper 92/5, Federal Reserve Bank of Atlanta.

15. McKenzie, Michael D. and R. Brooks (1997), "The Impact of Exchange Rate Volatility on German - US Trade Flows," Journal of International Financial Markets, Institutions and Money, 7.

16. Klein, M. (1990), "Sectoral Effects of Exchange Rate Volatility on United States Exports“. Journal of International Money and Finance, 9.

17. Adnan Kasman, Saadet Kasman (2005), "Exchange rate uncertainty in Turkey and its impact on export volume“, METU Studies in Development, 32 (June).

18. Krugman P., Obstfeld M., Melitz M. (2012), International Economics: Theory \& Policy.

19. To What Extent Do Exchange Rates and Their Volatility Affect Trade? The Case of Two Small Open Economies, China and New Zealand, TAD/TC/WP (2011).

20. Freund, Caroline and Martha Denisse Pierola, (2010), Export entrepreneurs: evidence from Peru, Policy Research Working Paper Series, \# 5407, The World Bank.

21. Haddad, Mona and Cosimo Pancaro (2010), Can Real Exchange Rate Undervaluation Boost Exports and Growth in Developing Countries? Yes, But Not for Long, Economic Premise, \# 20. World Bank.

22. Taglioni, Daria (2002), Exchange Rate Volatility as a Barrier to Trade: New Methodologies and Recent Evidences, Economie Internationale (CEPII), Quarter 1-2.

23. Ozturk, Ilhan (2006), Exchange Rate Volatility and Trade: A Literature Survey, International Journal of Applied Econometrics and Quantitative Studies, Vol. 3.

24. Auboin Marc and Ruta Michele (2011), The Relationship Between Exchange rates and International Trade: a Review of Economic Literature. Staff Working Paper ERSD-2011-17 27, WTO.

25. Rey S. (2006), Effective Exchange Rate Volatility and MENA countries Exports to the EU, Journal of Economic Development, 2, Volume 31, Number 2, December. http:// www.jed.or.kr/full-text/31-2/2.pdf 


\title{
Paradigm of Currency Exchange Fluctuations and Foreign Trade Dynamics
}

https://doi.org/10.35945/gb.2016.02.003

\author{
Mikheil Tokmazishvili \\ Doctor of Economic Sciences, Professor St. Andrew the First-Called Georgian \\ University of the Patriarchate of Georgia, Ass. Professor Ivane Javakhishvili Tbilisi \\ state University
}

Key words: foreign exchange, foreign trade, monetary policy, trade policy, Georgia

$\mathrm{T}$

his paper focuses on the currency rate changes and the impact of the cur-

rency fluctuations on trade flows. Through the empirical estimation of trade relations and exchange rate fluctuations, it is concluded, that the frequency of the foreign exchange fluctuations and the currency cycles were not reflected on Georgia-Turkey trade relations sufficiently. Trade flows were relatively dynamically. This case does not fully correspond to the widespread opinion according to which the exchange rate instability has a negative impact on trade relations, creates uncertainty and gives rise to the adverse effects of the expectations of business activity.

Impact of the exchange rate fluctuations on the countries' trade relations changes in the short and long term has different characteristics. Explanation of the export-import changes in short and medium term through the national currency exchange rate cycles does not show the correlation between them; and in the long-term - the national currency devaluation effect on dynamics of the trade relations was very slow. Foreign trade was sustainable, and the country's national currency fluctuations did not have a significant role in the trade changes. Moreover, impact of the exchange rate on the ratio of export and import and national currency appreciation has not led to the deterioration of export conditions. 